\title{
Sol-Gel Synthesis and Characterization of Microporous Silica Membranes I: SAXS Study on the Growth of Polymeric Structures
}

\author{
Balagopal N. Nair, ${ }^{1}$ Wilma J. Elferink, KlaAs Keizer, and Henk VerweiJ \\ Inorganic Material Science, Faculty of Chemical Technology, University of Twente, P.O. Box 217, 7500 AE Enschede, The Netherlands
}

Received May 15, 1995; accepted August 22, 1995

\begin{abstract}
Silica-based microporous membranes can be successfully processed by sol - gel techniques. H owever tailor making of such membranes is still not possible because of the unavailability of systematic studies on membrane oriented sol processing and characterization. The present series of papers aims to give a systematic understanding of silica polymeric growth, synthesis chemistry, and finally membrane characterization. Polymeric silica sols were prepared by hydrolysis and condensation of alkoxide. The fractal growth of the polymers during sol - gel transition is monitored with the help of small angle X-ray scattering. Fractal dimensions and radii of gyration of sols and gels with different synthesis chemistry are compared. The type of aggregation mechanism prevailing in these systems is identified as cluster - cluster aggregation with reaction limitations during synthesis and reaction and diffusion limitations during aging. $\odot 1996$ Academic Press, Inc.
\end{abstract}

Key Words: membrane; fractal; SAXS; silica.

\section{INTRODUCTION}

Microporous silica membranes are attractive materials for gas separation and membrane reactors. The synthesis and characterization of such membranes and their precursor materials have been dealt with by many authors for the past few years. Authors like Iler (1) and Scherer (2) have detailed descriptions of the whole sol-gel chemistry of silica and Yoldas (3) and Klein (4) have elaborated the sol-gel synthesis with emphasis to the wet gel characteristics. A number of articles were also published (5-9) about typical solgel silica membranes with porosity/permeation/separation behavior. Sol-gel silica binary systems for membrane synthesis were also dealt with (10). Even with all these studies, there are no selection rules available for tailor making microporous membranes.

It is the aim of the present authors to present a more detailed account of the sol-gel synthesis of silica along with characteristics of the resulting membranes, in a series of articles of which this is the first. The second part of this

\footnotetext{
${ }^{1}$ To whom correspondence should be addressed at Advanced Polymer Laboratory, Japan High Polymer Center, Yanagibashi 2-22-13, Taito-ku, Tokyo 111, Japan.
}

series (11) will present the synthesis chemistry of silica sols to make unsupported membranes with predictable pore size/ porosity. This article aims to give a detailed account of small angle X-ray scattering of silica sols and further calculations of fractal dimensions and monomer/polymer sizes. Fractal dimensions are compared with typical simulated aggregates from literature $(12,13)$.

Synthesis of silica polymers by the sol-gel process involve the hydrolysis and condensation reactions of starting alkoxides.

$$
\begin{aligned}
& \text { hydrolysis } \rightarrow \\
& \equiv \mathrm{Si}-\mathrm{OR}+\mathrm{H}_{2} \mathrm{O} \rightleftharpoons \equiv \mathrm{Si}-\mathrm{OH}+\mathrm{ROH} \\
& \leftarrow \text { esterification } \\
& \equiv \mathrm{Si}-\mathrm{OR}+\mathrm{HO}-\mathrm{Si} \equiv \rightleftarrows \equiv \mathrm{Si}-\mathrm{O}-\mathrm{Si} \equiv+\mathrm{ROH} \\
& \leftarrow \text { alcoholysis }
\end{aligned}
$$

$$
\begin{gathered}
\text { water condensation } \rightarrow \\
\equiv \mathrm{Si}-\mathrm{OH}+\mathrm{HO}-\mathrm{Si} \equiv \underset{\leftarrow \text { hydrolysis }}{\rightleftharpoons} \equiv \mathrm{Si}-\mathrm{O}-\mathrm{Si} \equiv+\mathrm{H}_{2} \mathrm{O} \\
\leftarrow \text { hy }
\end{gathered}
$$

Microporous structures are generally made available by acid catalysis of these reactions. Hydrolysis will then take place through a fast protonation of the alkoxide, followed by condensation to siloxane bond. By controlling the synthesis conditions and reactant concentrations the sol-gel morphology can be changed (2). This is essentially achieved by the control of rates of hydrolysis and condensation by changing the amount of water or catalyst used. The structure of the resulting polymers can change from more or less linear to weakly branched polymers. The morphology of the resulting gels depends on the structure of these polymers and to a better extent the efficiency of packing.

For the formation of microporous membranes, the ability of the polymers to interpenetrate must be considerable. Short branched linear polymers are the best in this regard. However, the probability is higher to form a dense structure when 
the interpenetration becomes very high. On the other hand highly branched systems can lead to inefficient packing with resulting mesoporosity of the gel. The branching and interpenetration of silica polymers can very well be explained with the help of fractal concepts (12).

Fractal objects possess dilation symmetry compared to translation or rotation symmetry of ordinary Euclidean objects (14). Fractals can be characterized by a dimension (Hausdorff dimension) which can take noninteger values to represent geometrical relationships. Silica polymeric systems grown by random processes are fractal objects and can be represented by a mass fractal dimension $\left(D_{\mathrm{f}}\right)$ related to mass $M$ and size $R$. $\left(M \sim R^{D_{\mathrm{f}}}\right)$. $D_{\mathrm{f}}$ has a limiting value of three, where the object is Euclidean. As the fractal dimension decreases the structure will look sparser, giving essentially a linear configuration at $D_{\mathrm{f}}=1(15)$. The tendency of fractal systems to interpenetrate is inversely related to the mean number of intersections given by

$$
M_{1,2} \propto R^{D_{\mathrm{f} 1}+D_{\mathrm{f} 2}-3} .
$$

As can be seen from the equation, smaller fractal dimensions encourage interpenetration (16).

Fractal dimensions of sols are hence found to be an important parameter. $D_{\mathrm{f}}$ of these polymers can be measured in a number of ways, for instance by light or neutron scattering. Small angle X-ray scattering (17) with synchrotron radiation is perfectly suitable for this analysis. A diffraction experiment leads to a scattering intensity $I$ as a function of the modulus of the scattering vector $Q$, defined as

$$
Q=\frac{2 \pi}{\lambda} \cdot \sin (2 \theta),
$$

where $\lambda$ is the wave length of radiation used and $2 \theta$ is the scattering angle.

The mathematical concept of fractality is valid for an infinite range of similitude. However as Mandelbrot himself puts it (14) for physical systems the occurrence of cutoff values is an unquestionable must. For mass fractal objects these correspond to the macroscopic apparent density $\left(\rho_{\mathrm{a}}\right)$ and the constant density of the elementary particle $\left(\rho_{\mathrm{s}}\right)$. In the polymeric systems we deal with $\xi$, the correlation length at which Euclidean behavior is observed, and $r_{0}$, the radius of the primary unit, represent these cutoff values. When the size of the polymer is in between these cutoff values fractal behavior is observed (18).

In a typical scattering curve $(\log I$ vs $\log Q)$ three regions can be identified: the Guinier region, above upper cutoff size (lower cutoff $Q$ value), the Porod region, below lower cutoff size, and the intermediate fractal region. The cutoff $Q$ values from fractal to Guinier region approximates $\xi(=1 / Q)$ and fractal to Porod region $r_{0}$ by an inverse relationship (10).
TABLE 1

Composition of Synthesis Mixtures

\begin{tabular}{cll}
\hline Sample & \multicolumn{1}{c}{$r_{\mathrm{a}}$} & $r_{\mathrm{w}}$ \\
\hline 2X & 0.17 & 6.4 \\
$\mathrm{X}$ & 0.085 & 6.4 \\
W13 & .1275 & 8 \\
W8 & .02125 & 9.6 \\
W9 & .02125 & 12.8 \\
\hline
\end{tabular}

The upper cutoff length $\xi$ can be correlated with the radius of gyration $\left(R_{\mathrm{g}}\right)(18)$.

From the fractal range, where the intensity has a power law relation, the dimension $D_{\mathrm{f}}$ can be calculated as the negative slope of the $\log I$ vs $\log Q$ curve.

$$
I \propto Q^{-D_{\mathrm{f}}} \quad\left(1 / \xi \ll Q \ll 1 / r_{0}\right)
$$

However a number of problems can in fact make the assessment of all these parameters tricky. If the concentration of the polymer is high, underestimation of $R_{\mathrm{g}}$ will result because of entanglement. Near the percolation threshold the estimation of $R_{\mathrm{g}}$ is difficult too. Calculation of $D_{\mathrm{f}}$ must theoretically be done on a minimum fractality range of one decade $\left(\xi / r_{0}\right.$ $=10)$, which is difficult to find in most of the measured systems (18). Even with all these difficulties, estimation of the fractal dimension and cutoff radii may give a good indication of the structure of the gel.

\section{MATERIALS AND METHODS}

Polymeric sols were prepared by hydrolysis and condensation of tetraethyl-orthosilicate (TEOS, Merck, p.a. grade) in ethanol with $\mathrm{HNO}_{3}$ as catalyst. A mixture of $\mathrm{HNO}_{3}$ (Merck, p.a. grade) and water was carefully added, using a dropping funnel, to a mixture of ethanol (ethanol absolute, Merck, p.a. grade) and TEOS under vigorous stirring. The reaction mixture was then refluxed for $3 \mathrm{~h}$ at $65 \pm 5^{\circ} \mathrm{C}$ under stirring. The amount of ethanol added was kept constant during the present experiments. The compositions of the sols were TEOS:ethanol: $r_{\mathrm{a}}: r_{\mathrm{w}}=1: 3.8: r_{\mathrm{a}}: r_{\mathrm{w}}$, in which $r_{\mathrm{a}}$ is the molar ratio of $\mathrm{HNO}_{3}$ to TEOS and $r_{\mathrm{w}}$ is the molar ratio of $\mathrm{H}_{2} \mathrm{O}$ to TEOS. In the experiments in which the effect of $\mathrm{HNO}_{3}$ (Figs. 6 and 8) was measured, $r_{\mathrm{w}}$ was kept constant at 6.4. All the other compositions of sols and the used codes are presented in Table 1. Unless mentioned otherwise, sols in this paper correspond to composition $\mathrm{X}$ in Table 1.

The sols prepared had a concentration of $2 M$ Si. Part of the sol was diluted with ethanol to $0.2,0.1$, and $0.05 \mathrm{M}$. The prepared sols were kept in closed glass bottles at room temperature for aging. Unsupported membranes for porosity measurements were prepared by drying a $0.1-M$ sol in a Laminar flow cupboard. Dried samples were calcined at $400^{\circ} \mathrm{C}$ for $3 \mathrm{~h}$. Nitrogen adsorption isotherms were plotted 
TABLE 2

Change in $D_{\mathrm{f}}$ and $\mathrm{G}$ \& Porosity with Reactant Concentration

\begin{tabular}{llccc}
\hline Sample & \multicolumn{1}{c}{$r_{\mathrm{a}}{ }^{a}$} & $r_{\mathrm{w}}{ }^{b}$ & $\varepsilon^{c}$ & $D_{\mathrm{f}}{ }^{d}$ \\
\hline 2X & 0.17 & 6.4 & 27.5 & 1.53 \\
W13 & 0.1275 & 8 & 24.6 & 1.36 \\
W9 & 0.02125 & 12.8 & 1.33 & 1.16 \\
W8 & 0.02125 & 9.6 & 0 & 1.00 \\
\hline
\end{tabular}

${ }^{a} r_{\mathrm{a}}$ is the molarity of $\mathrm{H}+$ in $1 \mathrm{~mol}$ of TEOS.

${ }^{b} r_{\mathrm{w}}$ is the molarity of water in $1 \mathrm{~mol}$ of TEOS.

${ }^{c} \varepsilon$ is the $\%$ porosity of the calcined gel.

${ }^{d} D_{\mathrm{f}}$ is the mass fractal dimension.

at liquid nitrogen temperatures on a Micromeritics ASAP 2000 and porosity was calculated assuming a skeletal density of $2.2 \mathrm{~g} / \mathrm{cm}^{3}$ for silica.

The small angle $\mathrm{X}$-ray scattering experiments were performed using synchrotron radiation (X-ray wavelength $\lambda=$ $1.54 \AA$ ) at the noncrystalline diffraction beam line 8.2 of the SERC Synchrotron Radiation Source (SRS) in Daresbury (UK). The scattered X-ray intensities were recorded using a quadrant detector with camera lengths of 2 and $0.6 \mathrm{~m}$ (Fig. 9 and Table 2). The used windows were mostly Mylar but Kapton for the results presented in Figs. 6 and 8. The obtained spectra were corrected for background and parasitic scattering.

Most of the sol samples used for the results presented in Figs. 1-8 were prepared in our laboratory and aged under ambient conditions until measurement. Fresh sols and sols with aging times less than $50 \mathrm{~h}$ were prepared in the Daresbury Laboratory in-campus facility. Sols for Fig. 9 and Table 2 were prepared in our laboratory a few weeks before measurements but kept at $-20^{\circ} \mathrm{C}$ until measurement. This made the degree of condensation in these samples quite low (however, absolute comparison between $D_{\mathrm{f}}$ of these sols and a fresh sol can be error prone). The molarity of the measured sol is $2 M$ unless otherwise mentioned.

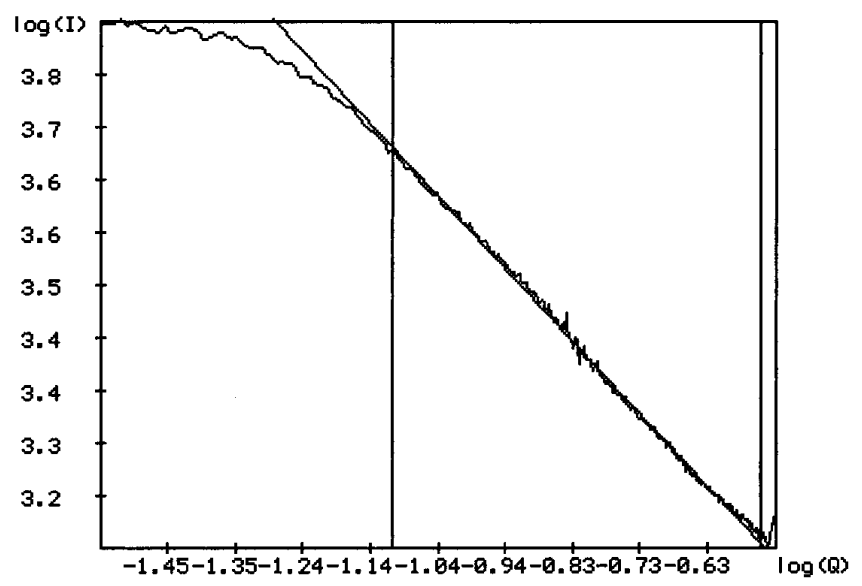

FIG. 1. SAXS spectra of freshly prepared sol X. $D_{\mathrm{f}}=0.963$.

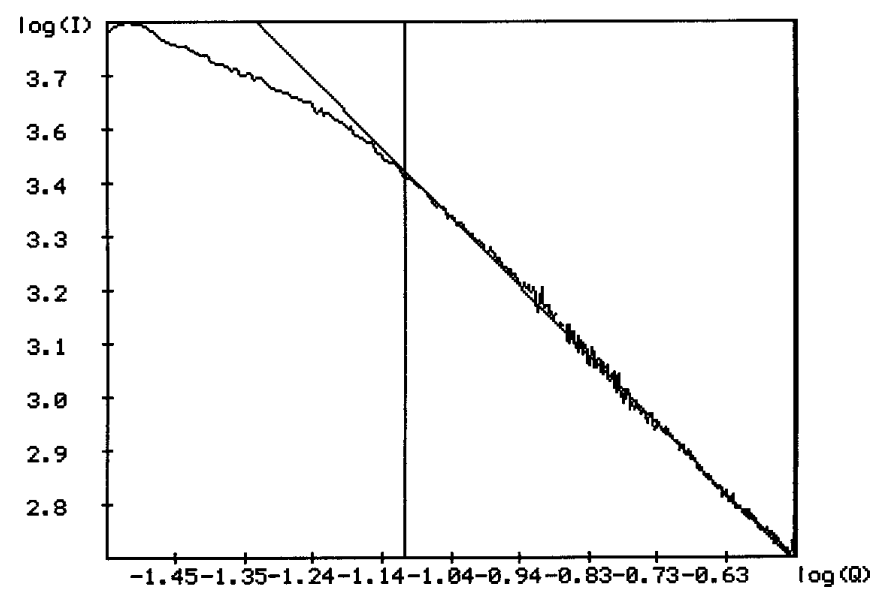

FIG. 2. SAXS spectra of sol $X$ after 6 days. $D_{\mathrm{f}}=1.397$.

\section{RESULTS}

The aggregation process in silica was monitored with small angle $\mathrm{X}$-ray scattering. A standard silica sol (X) was prepared at $65 \pm 5^{\circ} \mathrm{C}$ with $3 \mathrm{~h}$ refluxing as described in the Experimental. The slope of the $\log I$ vs $\log Q$ curve in the intermediate region was measured as -0.41 after $1 \mathrm{~h}$ and -0.63 after $2 \mathrm{~h}$ of synthesis. The structure is growing at this stage. By the end of refluxing $(3 \mathrm{~h})$ morphological growth became almost stable and indications of linear connected fractals have appeared with fractal dimension around 0.96 as in Fig. 1. These sols were then aged in closed bottles at room temperature. Figure 2 shows the scattering curve of the sol aged for 6 days. A fractal dimension of 1.4 was measured. The radius of gyration at this level of branching is calculated as approximately $15 \AA$. The increase in fractal dimension and radius of gyration with time is shown in Fig. 3. It can be seen that after a preliminary induction period of around $100 \mathrm{~h}$ the fractal dimension increases faster until it reaches a plateau. A final fractal dimension of 2.15 was measured after 8 months aging. $R_{\mathrm{g}}$, however, could be measured only up to $36 \AA$. The fractal image of a gelated system ( 6 months aged) is shown in Fig. 4. It can be seen that both Porod and Guinier regions were out of the measured window. Figure 5 presents the change in fractal dimension during

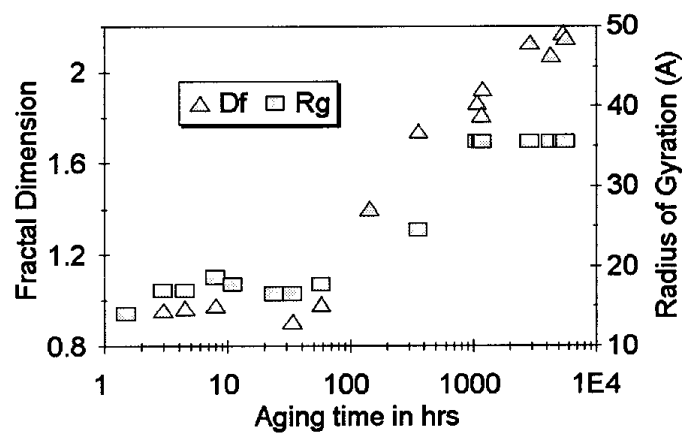

FIG. 3. Change in fractality of sol $X$ with aging. 


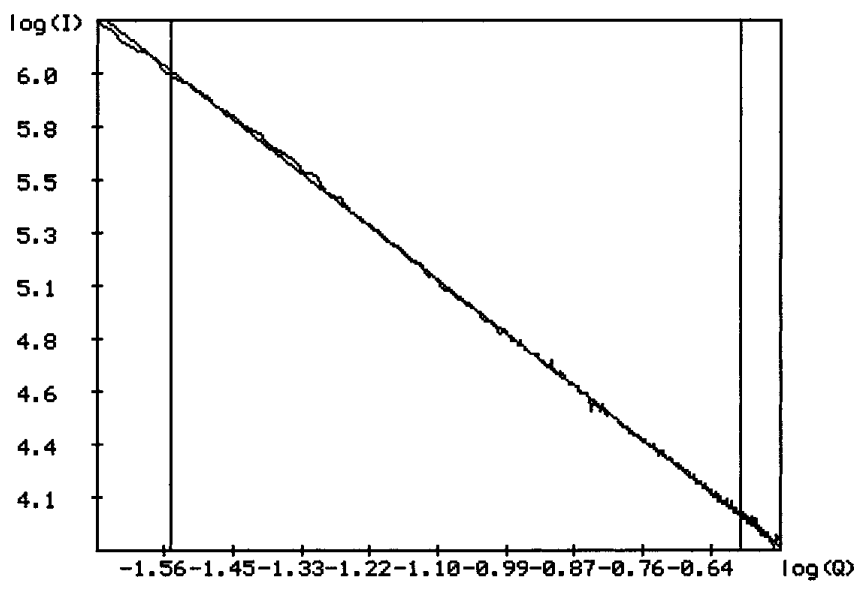

FIG . 4. SAXS spectra of silica gel. $D_{\mathrm{f}}=2.074$.

aging because of concentration differences of the sol. The $2 M$ sol increases its fractal dimension with aging time faster than the $0.05 M$ sol.

As can be seen in Fig. 6 the fractal dimension of the sol is also a function of the catalyst concentration during synthesis. Typical results of approximately 40-day aged sols $(\sim$ gel $)$ are shown in Fig. 6 (constant $\left.r_{\mathrm{w}}=6.4\right)$. The fractal dimension and radius of gyration were observed to increase with catalyst concentration in the sol. Table 2 (measured with a $0.6 \mathrm{~m}$ camera on the $0.5 \mathrm{M}$ sol) also shows the increase in fractal dimensions of sols (kept at $-20^{\circ} \mathrm{C}$ until measurement) with an increase in reactant concentrations. As can be seen, an increase in catalyst (W9 $\rightarrow$ W13 $\rightarrow 2 \mathrm{X}$ ) concentration (even with a decrease in water concentration) increases $D_{\mathrm{f}}$. Roughly the same is true for an increase in water content (W8 $\rightarrow$ W9). From the table the dominant effects of change in acid concentration over that of water is apparent. These effects are further discussed elsewhere (11). The porosity of the resulting calcined gel also increases in the same fashion as shown in Fig. 7 and Table 2. The nitrogen adsorption isotherms in Fig. 7 show samples W13 and $2 \mathrm{X}$ to be microporous (type 1 ) and the others nearly dense. The porosity of the samples as in Table 2 were calculated

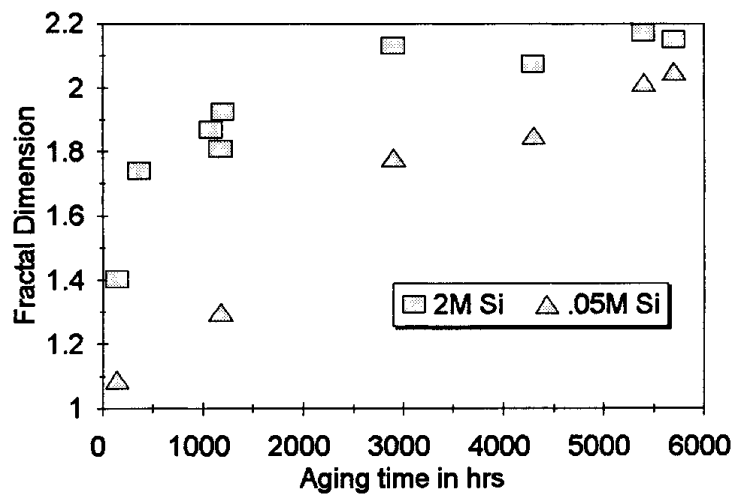

FIG. 5. Effect of dilution on fractal growth of silica polymers.

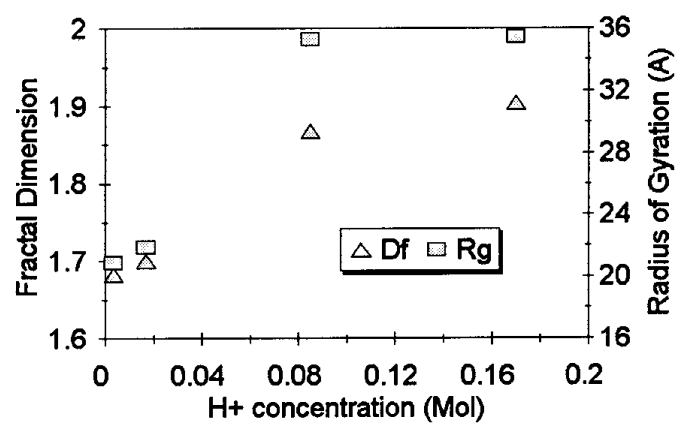

FIG. 6. Effect of $\mathrm{H}^{+}$concentration (constant $r_{\mathrm{w}}=6.4$ ) on the gel structure.

from the total adsorbed volume calculated by the isotherm intercept method (19). More details of porosity changes with different concentrations of water and catalyst will be given in the companion paper (11).

The effect of concentration of the sol on the fractal dimension is shown in Fig. 8. The 0.2, 0.1, and $0.05 M$ sols showed smaller fractal dimensions than the $2 M$ sol at all catalyst concentrations. Apparently low and moderate concentrations of the sol give nearly the same structure for the gel.

The camera length used for most of the analyses was inadequate to obtain large $Q$ values, so a number of measurements were performed (Table 2 and Fig. 9) on a short camera $(0.6 \mathrm{~m})$. A typical scattering curve is shown in Fig. 9. A sol with catalyst concentration $0.17 \mathrm{~mol}$ (sample $2 \mathrm{X}$ in Table 1) showed a clear transition from the Porod to fractal region at $2.5 \AA$. Most of the sols showed a transition around this value and hence the primary unit can be assumed to be of around $5 \AA$ in diameter.

\section{DISCUSSION}

Hydrolysis and condensation reactions in silicon alkoxides depends on reactant concentrations as well as conditions such as the reaction temperature. Higher temperatures generally lead to more branched structures which, in turn, yield

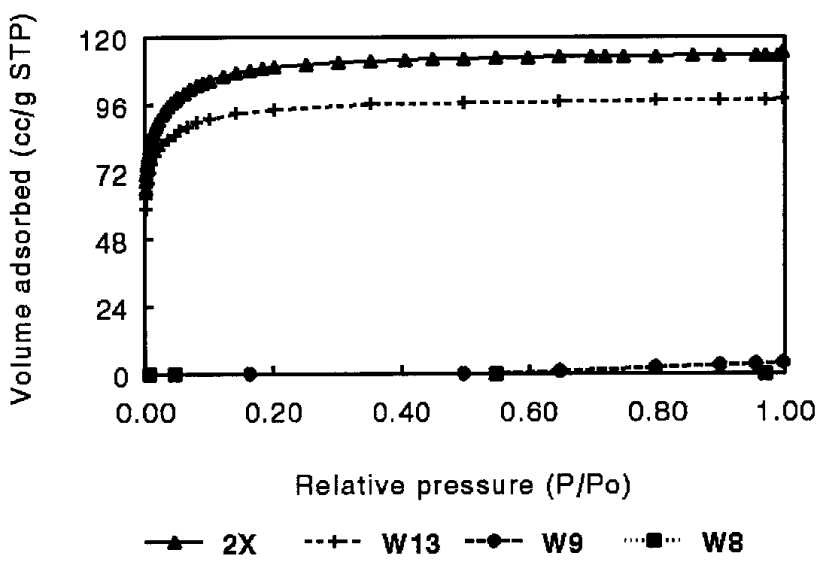

FIG. 7. Nitrogen adsorption isotherms of calcined silica gels. 


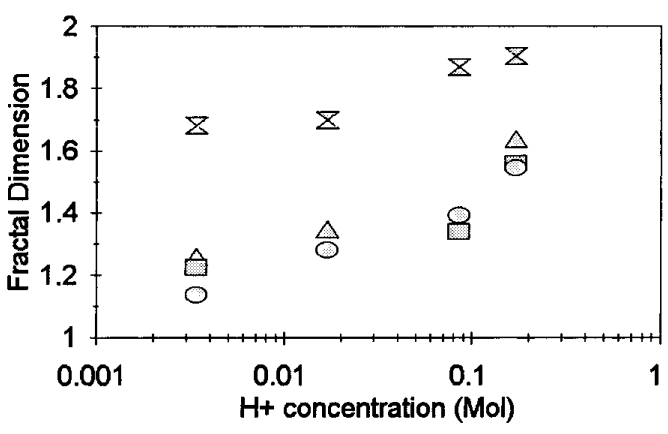

$\mathrm{Z} 2 \mathrm{MSi} \triangle .2 \mathrm{MSi} .1 \mathrm{M} \mathrm{Si} \odot .05 \mathrm{M} \mathrm{Si}$

FIG. 8. Effect of dilution on fractal growth of silica polymers with different $r_{\mathrm{a}}$ (constant $r_{\mathrm{w}}=6.4$ ).

porous materials (2). The effect of reactant concentrations on the gel structure is detailed elsewhere (11). Another variable which can change the pore structure of the gel is aging (6). Figures 1-5 essentially indicate these developments in gel structure with time under ambient conditions. The sol which showed a slope of 0.4-0.6 during the growing stage has developed more or less linear connected morphology by the end of synthesis with a fractal slope of 0.96 . A fractal dimension of 1 theoretically represents linear connected fractal polymers (15). Because of the possibility of interpenetration these polymers can give rise to well-packed membranes. Consequently smaller pore sizes or porosities are realized.

Aging of the sol as shown in Fig. 3 is rather slow at the beginning. The reason for this is not very clear at this moment. The fractal dimension remains constant for the first few decades of hours. This induction period can be interpreted as ordering of aggregates before actual gelation takes place. If geometrical ordering in fact takes place, configuring the coiling and or spatial position of polymers because of dipolar effects, it is not surprising that $D_{\mathrm{f}}$ remains constant as is roughly the trend.

After the induction period the system aggregates much faster and gelates to reach almost constant fractality. A final fractal dimension of 2.15 was observed after 8 months. This value is higher than expected for a classical tip-tip aggregate. However Beelen et al. (13) has recently reported simulated fractal dimensions of 2.1 on DLCCA (diffusion limited cluster-cluster aggregation) aggregates with reaction constraints too. Our results show the following trend. During synthesis, reaction chemistry, as is going to be shown, influences condensation rates more than diffusion (RLCCA) and during aging, diffusion at low temperature will be low enough to be rate determining (DLCCA/RLCCA).

The fractal value of 2.15 measured at the gelation end may be exaggerated because of ensemble (20) effects. The radius of gyration also is difficult to measure once the system reaches the percolative threshold. As can be seen in Fig.
4 the transition between the Guinier and fractal region is immeasurable in the window of present $Q$ values.

As can be seen in Fig. 5 dilution can also affect the kinetics of aggregation during aging. The $0.05 \mathrm{M}$ sol reaches gelation in a slower rate. The difficulty in reaching the percolation threshold at this dilution is the reason for this delay. The dominant effect of diffusion limitations on the aggregation is clear. If RLCCA was the only process, both systems had to show a similar fractal geometry throughout the growth. On dilute systems diffusion limitations are more dominant and hence the delay. The more or less similar final $D_{\mathrm{f}}$ values ( 2.05 of the diluted system against 2.15 of the $2 \mathrm{M}$ sol), however, show the effect of reaction constrains on the final gel structure.

Figure 6 and Table 2 present a systematic picture of fractality changes because of synthesis chemistry variations. $D_{\mathrm{f}}$ and $R_{\mathrm{g}}$ increases with catalyst concentration and water concentration in the reactant mixture. The increase in $\mathrm{H}^{+}$concentration increases the condensation rates resulting in polymers with a higher degree of branching. The micropore volume of the dried gels also follows the same sequence as that of the fractal dimension of the sols (Table 2) and wet gels (Fig. 6). It has to be noted that gels are dried open, compared to closed aging of sol for the aging experiments, creating loss of alcohol and saturation of water during initial drying ( simultaneous to condensation) periods. Even with this change in the kinetics of condensation, it is shown that the initial fractal objects represent the final morphological features of the gel to a good extent.

Another important parameter which can change morphology is temperature. As already said the syntheses reported here are all done at $65^{\circ} \mathrm{C}$. This is the reason for the difference between the starting fractal dimensions of 0.96 to 1.3 between this work and that of de Lange et al. (10), even though the composition was nearly the same. However the typical leap in fractality in the case of de Lange et al., where in a nonscattering synthesis the mixture suddenly turns into

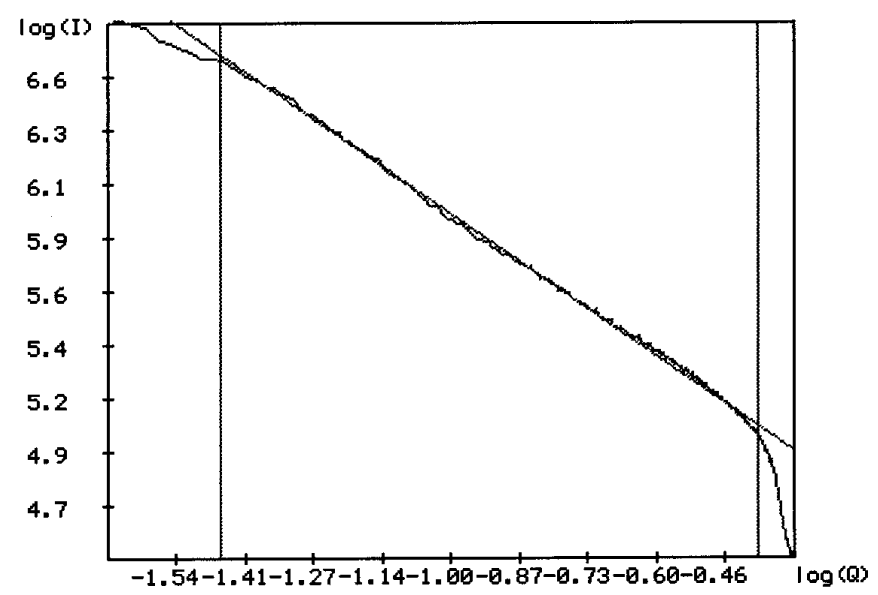

FIG. 9. SAXS spectra of silica sol $2 X$ showing Porod and Guinier regions. $D_{\mathrm{f}}=1.533, R_{\mathrm{g}}=28.5 \AA, r_{0}=2.5 \AA$. 
an aggregate with a fractal dimension 1.3 , cannot be very well understood. The $2 \mathrm{X}$ sol reported in Table 2 also has a high starting fractal dimension of 1.53. In the case of $2 \mathrm{X}$ sols as well as in the case of de Lange's results the hydrolysis and condensation reactions are faster, because of the higher amount of catalyst used and the higher synthesis temperature, respectively. This large deviation of $D_{\mathrm{f}}$ (compared to sol $\mathrm{X}$ ) by the end of synthesis clearly shows the reaction limitations on polymeric growth during synthesis. This is exactly why the branching and hence fractal dimension of the polymers vary with catalyst concentration as shown in Table 2.

Again dilution has significant effects on the formation of aggregates (Fig. 8). The similarity of $D_{\mathrm{f}}$ values of dilute sols $(0.2,0.1$, and $0.05 M)$ and the large difference with those of the $2 M$ sol clearly show the presence of an early percolative threshold, caused by volume restrictions to ordering, for highly concentrated sols.

These results have important implications on membrane synthesis. The formation of an early percolated structure, particularly at the surface, can hinder the formation of crackfree membranes. Therefore, concentrated systems like the 2 $M$ sol are not the best choice for dip solutions to make supported membranes. As mentioned before typically $0.1 M$ diluted systems have a slower rate of morphological development, which make them possibly more suitable for microporous membrane synthesis. The proximity of $D_{\mathrm{f}}$ values of gelated dilute systems show indeed the possibility of making similar membranes over a wide range of dilutions. The importance of keeping drying rate small is clear. The formation of early percolated structures like in the case of concentrated sol can otherwise result. This is particularly important when freshly prepared sols with a low degree of branching, and hence high interpenetration, are used.

Finally, Fig. 9 presents the more classical scattering spectra with Porod, fractal, and Guinier regions. $r_{\mathrm{o}}$ values can be calculated to be in the region of $2.5 \AA$. This goes along with the findings of de Lange (10) and Kamiyama (21). However, contrary to Kamiyama's results the changes in $r_{\mathrm{o}}$ with synthesis chemistry was not that statistically significant.

The mechanism of aggregation in the silica sol-gel can be identified as a combination of DLCCA and RLCCA. The fractal results are complimentary with the porosity results obtained. It can be realized that the evaluation of fractal dimension and radius of gyration approximates the porosity situation in dried unsupported membranes.

\section{SUMMARY}

1. The fractal dimension and radius of gyration of silica polymers increases during the sol-gel transition.

2. Increasing reaction rates during synthesis can increase the degree of branching of the resulting polymers and hence increases the porosity of the resulting membrane.

3. More or less linear polymers can be realized using moderate reaction rates.

4. The aggregation mechanism can be identified as RLCCA with DLCCA, also during aging, leading to final gel fractal dimensions of 2.15.

5. It is advisable to use dilute dip solutions as well as reasonably low drying rates in order to realize membranes with consistent texture and morphology.

\section{ACKNOWLEDGMENTS}

The authors thank R. S. A. de Lange and R. M. de Vos for experimental and theoretical assistance. W. Bras (NWO-SERC/SRS, Daresbury) is acknowledged for his kind help during the beam time. Professor A. J. Burggraaf is thanked for the stimulating discussions. G. M. H. van de Velde and $\mathrm{H}$. Karrenbeld are acknowledged for their help during data treatment. NWO and European community are specially thanked for financial assistance.

\section{REFERENCES}

1. Iler, R. K., "The Chemistry of Silica." Wiley, New York, 1979.

2. Brinker, C. J., and Scherer, G. W., "Sol-Gel Science: The Physics and Chemistry of Sol-Gel Processing." Academic Press, London, 1990.

3. Yoldas, B. E., J. Mater. Sci. 14, 1843 (1979).

4. Klein, L. C., Ann. Rev. Mater. Sci. 15, 227 (1985).

5. Julbe, A., Guizard, C., Larbot, A., Cot, L., and Giroir-Fendler, A., J. Membr. Sci. 77, 137 (1993).

6. de Lange, R. S. A., "Microporous Sol-Gel Derived Ceramic Membranes for Gas Separation"' University of Twente, The Netherlands, 1993.

7. Maier, W. F., Tilgner, I-C., Wiedorn, M., Ko, H-C., Ziehfreund, A., and Sell, R., Adv. Mater. 5, 730 (1993).

8. Brinker, C. J., Ward, T. L., Sehgal, R., Raman, N. K., Hietala, S. L., Smith, D. M., Hua, D-W., and Headley, T. J., J. Membr. Sci. 77, 165 (1993).

9. Uhlhorn, R. J. R., Keizer, K., and Burggraaf, A. J., J. Membr. Sci. 66, 271 (1992)

10. de Lange, R. S. A., Hekkink, J. H. A., Keizer, K., and Burggraaf, A. J., J. Non-Cryst. Solids 191, (1995).

11. Elferink, J. W., Nair, B. N., de Vos, R. M., Keizer, K., and Verweij, H., submitted for publication, (1995).

12. Schaefer, D. W., and Keefer, K. D., Mater. Res. Soc. Symp. Proc. 72, 277 (1986).

13. Beelen, T. P. M., Dokter, W. H., Van Garderen, H. F., and Van Santen, R. A., Adv. Colloid Interface Sci. 50, 23 (1994).

14. Mandelbrot, B. B., “The Fractal Geometry of Nature.' Freeman, New York, 1983.

15. Martin, J. E., and Hurd, A. J., J. Appl. Crystallogr. 20, 61 (1987).

16. Brinker, C. J., Sehgal, R., Hietala, S. L., Deshpande, R., Smith, D. M., Loy, D., and Ashley, C. S., J. Membr. Sci. 94, 85 (1994).

17. Glatter, O., and Kratky, O., "'Small Angle X-ray Scattering." Academic Press, London, 1982.

18. Zarzycki, J., J. Non-Cryst. Solids 121, 110 (1990).

19. De Lange, R. S. A., Keizer, K., and Burggraaf, A. J., J. Porous Mater. 1, 139 (1995).

20. Martin, J. E., and Wilcoxon, J., Mater. Res. Soc. Symp. Proc. 180, 199 (1990).

21. Kamiyama, T., Mikami, M., and Suzuki, K., J. Non-Cryst. Solids 150, 157 (1992). 